\title{
Electronic Structure of the Aqueous Vanadyl Ion Probed by 9 and 94 GHz EPR and Pulsed ENDOR Spectroscopies and Density Functional Theory Calculations
}

\author{
Christopher V. Grant, ${ }^{\dagger}$ William Cope, ${ }^{\dagger}$ James A. Ball, ${ }^{\dagger}$ Guenter G. Maresch, \\ Betty J. Gaffney, William Fink, ${ }^{\dagger}$ and R. David Britt*, \\ Department of Chemistry, University of California, Davis, California 95616, and Institute of Molecular \\ Biophysics, Florida State University, Tallahassee, Florida 32306
}

Received: June 28, 1999; In Final Form: September 20, 1999

\begin{abstract}
The aqueous vanadyl ion $\left(\left[\mathrm{VO}\left(\mathrm{H}_{2} \mathrm{O}\right)_{5}\right]^{2+}\right)$ has been investigated by X-band EPR, $94 \mathrm{GHz} \mathrm{W}$-band EPR, and ESE-ENDOR. These experiments reveal information about the hyperfine $\left(\left|A_{x x}\right|=208.5 \mathrm{MHz},\left|A_{y y}\right|=208.5\right.$ $\left.\mathrm{MHz},\left|A_{z z}\right|=547.0 \mathrm{MHz}\right)$, and nuclear quadrupole coupling $\left(\left|e^{2} q Q\right|=5.6 \mathrm{MHz}\right)$ of the ${ }^{51} \mathrm{~V}$ nucleus. The measured nuclear quadrupole coupling parameters are compared to values determined by density functional theory calculations $\left(\left|e^{2} q Q\right|=5.2 \mathrm{MHz}\right)$. These theoretical calculations illustrate that axial ligands and molecular distortions can alter the magnitude of the nuclear quadrupole interaction.
\end{abstract}

\section{Introduction}

Recent interest in vanadium spectroscopy and solution chemistry has been motivated by the discovery of vanadium containing enzymes, ${ }^{1,2}$ the use of the vanadyl ion as a spin probe, ${ }^{3,4}$ and the insulin mimetic ability of vanadium in mammals. ${ }^{5-7}$ EPR spectroscopy has been used extensively in the study of vanadyl with biologically relevant ligands, ${ }^{4}$ however, EPR alone offers little insight into the nature of ligands binding trans to the oxo group of the vanadyl ion and little information about ligand geometry. ${ }^{3,8}$ Information about axial ligands and ligand geometry is necessary for fully understanding the interaction of the vanadyl ion with biological molecules. Therefore, we have been working toward the development of ENDOR spectroscopy as an additional structural probe of the $\mathrm{V}(\mathrm{IV})$ vanadyl ion $\left(\mathrm{VO}^{2+}\right)$, allowing for the measurement of the nuclear quadrupole interaction which is sensitive to these structural features. ${ }^{9}$

At moderately low $\mathrm{pH}$, the aqueous vanadyl ion exists as $\left[\mathrm{VO}\left(\mathrm{H}_{2} \mathrm{O}\right)_{5}\right]^{2+}$. This species has been studied extensively by EPR techniques in powders, ${ }^{10-12}$ doped single crystals, ${ }^{13-16}$ a polymer matrix, ${ }^{17}$ and frozen solutions. ${ }^{18,19}$ The molecular structure was first elucidated by Atherton and Shackleton by EPR and ENDOR spectroscopies. ${ }^{15}$ The hyperfine components of all proton classes of the five coordinating water molecules were determined for the vanadyl ion doped into a single crystal of $\mathrm{Mg}\left(\mathrm{NH}_{4}\right)_{2}\left(\mathrm{SO}_{4}\right)_{2} \cdot \mathrm{H}_{2} \mathrm{O}$. Many studies of the approximately axial vanadyl spin system have concluded that the coincidence of the axis systems of the hyperfine and $\mathbf{g}$ matrices is high along the VO bond axis. ${ }^{12-15}$ Some of these studies have reported a slight displacement of the axis system of $\mathbf{A}$ with respect to $\mathbf{g}$ along the equatorial axis $\left(<25^{\circ}\right),{ }^{13,15}$ and others report high coincidence for the equatorial axis. ${ }^{12,16}$ In the case of true $C_{4 v}$ symmetry, coincidence of the $\mathbf{g}$ and $\mathbf{A}$ tensors is required, and the aqueous vanadyl ion seems to exhibit near coincidence of these interactions. This structurally well characterized vanadyl complex is useful for the comparison of matrix diagonalization

* Corresponding author.

$\dagger$ University of California, Davis.

$\doteqdot$ Florida State University. and perturbation theory analysis of ${ }^{51} \mathrm{~V}$ ENDOR data and is of manageable complexity for comparing these experimental results to calculated theoretical values of the nuclear quadrupole coupling constants calculated with ab initio density functional theory (DFT).

\section{Experimental Section}

The pulsed EPR/ENDOR spectrometer has been described previously. ${ }^{20,21}$ The pulsed ENDOR experiments were performed using the Davies pulse sequence. ${ }^{22}$ Relatively long microwave pulses (100 ns $\pi / 2$ microwave pulse duration) were used to allow for the best possible orientation selection. ${ }^{23}$ The $94 \mathrm{GHz} \mathrm{cw}$ EPR spectrometer and the X-band cw EPR spectrometer are Bruker models ELEXSYS E 600 and ECS106, respectively. The vanadyl samples were prepared from $\mathrm{VOSO}_{4} \cdot 2 \mathrm{H}_{2} \mathrm{O}$ at $\sim 5 \mathrm{mM}$ concentration in $\mathrm{H}_{2} \mathrm{O}$ solution containing $40 \%$ glycerol by volume as a cryoprotectant and titrated to approximately $\mathrm{pH}=$ 2 with $\mathrm{HCl}$.

$\mathrm{Ab}$ initio calculations utilizing the Gaussian-94 program package $^{24}$ were performed on pentaaquaoxovanadium(IV) and tetraaquaoxovanadium(IV). The program uses a density functional theory algorithm to calculate the electric field gradient at the vanadium nucleus. B3LYP hybrid functionals were employed to determine the electronic energy. Molecular geometries were optimized to an energy minimum on the potential surface. The $3-21 \mathrm{G}$ basis set was used for molecular geometry optimization and the $6-31 \mathrm{G}^{*}$ basis $\operatorname{set}^{25}$ for vanadium was used for all electric field gradient calculations.

\section{Magnetic Resonance Background and Theory}

The spin Hamiltonian for the vanadyl spin system (electron spin $S=1 / 2$, nuclear spin $I=7 / 2$ ) is constructed as follows, with electron Zeeman, hyperfine, isotropic nuclear Zeeman, and electric nuclear quadrupole terms, respectively:

$$
\mathrm{H}=\beta \vec{B} \cdot \mathbf{g} \cdot \vec{S}+\vec{S} \cdot \mathbf{A} \cdot \vec{I}-\beta_{n} g_{n} \vec{B} \cdot \vec{I}+\vec{I} \cdot \mathbf{P} \cdot \vec{I}
$$

The sign of the hyperfine interaction is assumed negative in this work. ${ }^{12}$ Within its principal axis system, the nuclear 
quadrupole interaction term of the hamiltonian, $\vec{I} \cdot \mathbf{P} \cdot \vec{I}$, can be written as ${ }^{26}$

$$
P_{\|}\left[\left\{I_{z}^{2}-(1 / 3) I(I+1)\right\}+(\eta / 3)\left\{I_{x}^{2}-I_{y}^{2}\right\}\right]
$$

where the traceless nuclear quadrupole tensor $\mathbf{P}$ is represented by the parameters $P_{\|}$and $\eta$ :

$$
\begin{gathered}
P_{\|}=(3 / 2) P_{z z}=3 e^{2} q Q /[4 I(2 I-1)]=3 e^{2} q Q / 84 \\
\quad(\text { for } I=7 / 2) \\
\eta=\left|\left(P_{x x}-P_{y y}\right)\right| / P_{z z}
\end{gathered}
$$

where $P_{x x}, P_{y y}$, and $P_{z z}$ are the diagonal elements of $\mathbf{P}, q$ is the electric field gradient along the principal axis (z-axis) of the largest field gradient, and $Q$ is the nuclear quadrupole moment $\left(-0.05 \times 10^{-24} \mathrm{~cm}^{2}\right){ }^{27}$ The asymmetry parameter $\eta$ is a measure of gradient asymmetry in the plane perpendicular to the $z$-axis and may range from zero for the case of axial symmetry to 1 in the fully rhombic limit. We have previously reported the details of a second-order perturbation theory analysis of ENDOR data for a series of $\mathrm{VO}$ complexes. ${ }^{9}$ The following analytical perturbation theory expression can be used for the estimation of $P_{\|}$:

$$
\begin{aligned}
\Delta v^{\prime \prime}(+7 / 2)-\Delta v^{\prime \prime}(-7 / 2)= & \Delta \Delta v_{ \pm}^{\prime \prime}(7 / 2)= \\
& 2 g_{n} \beta_{n}\left(B_{+7 / 2}-B_{-7 / 2}\right)-24 P_{\|}
\end{aligned}
$$

where $\Delta v^{\prime \prime}(+7 / 2)$ and $\Delta v^{\prime \prime}(-7 / 2)$ are the difference frequencies of the pairs of ENDOR transitions obtained at the $M_{I}= \pm 7 / 2$ parallel turning points (i.e., the $B_{ \pm 7 / 2}$ field positions for a given complex). The estimates of $P_{\|}$by perturbation theory in this work utilize the above expression. In this work we introduce a spectral simulation analysis of the ENDOR data that provides an improved estimate of $P_{\|}$using direct diagonalization of the spin Hamiltonian (eq 1) via a FORTRAN program of our own construction.

\section{Results}

EPR. Figures 1 and 2 display X-band and W-band frequency cw EPR spectra of the solvated $\mathrm{VO}^{2+}$ ion and spectral simulations utilizing direct diagonalization of eq 1 . The matrix diagonalization simulations involve diagonalizing the spin Hamiltonian for 12000 molecular orientations of the vanadyl ion with respect to the external magnetic field, where the electron $\mathbf{g}$ tensor establishes the molecular reference axis. The formalism used allowed for the rotation of the hyperfine and quadrupole matrices of eq 1 with respect to the molecular reference axis; however, all fits were achieved without rotating these interaction matrices. These matrix diagonalizations are performed at each field value and only those transition frequencies which are within a specified bandwidth of the resonant frequency (16 MHz for X-band, and $72 \mathrm{MHz}$ for W-band) contribute to the calculated spectrum. Simulations performed at the two disparate frequencies ensure accurate determination of both $\mathbf{g}$ and $\mathbf{A}$ matrices. The $95 \mathrm{GHz} \mathrm{W}$-band spectrometer allows for high resolution of $\mathbf{g}$ values and is thus useful for determining the extent to which the $\mathbf{g}$ matrix is axial. Both spectra are fit well with the following parameters: $g_{x x}=1.9792$, $g_{y y}=1.9772, g_{z z}=1.9335$, and $A_{x x}=-208.5 \mathrm{MHz}, A_{y y}=$ $-208.5 \mathrm{MHz}, A_{z z}=-547.0 \mathrm{MHz}$. This small amount of rhombicity in the $\mathbf{g}$ matrix does not allow for additional features to be resolved in the W-band EPR spectrum, giving rise to a

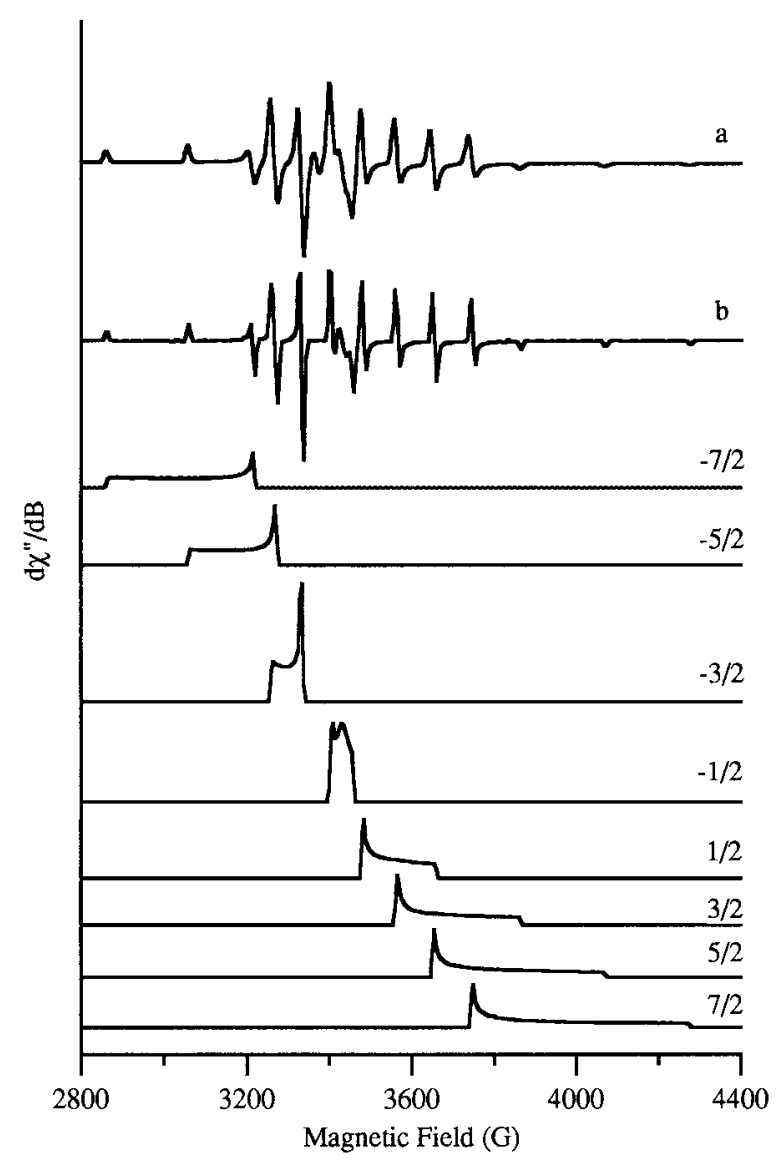

Figure 1. X-band EPR spectrum (a) of $\left[\mathrm{VO}\left(\mathrm{H}_{2} \mathrm{O}\right)_{5}\right]^{2+}$; trace (b) is the matrix diagonalization simulation. The additional traces show the eight individual $M_{I}=-7 / 2, \ldots,+7 / 2$ powder patterns. These traces are shown in absorption mode rather than as a derivative as in the cw-EPR spectrum for simplicity. Experimental conditions are as follows: $\mathrm{v}_{\mathrm{mw}}=$ $9.68 \mathrm{GHz}$; MW power $=\sim 25 \mathrm{~mW}$; modulation amplitude $=8.0 \mathrm{G}$; time constant $=20.48 \mathrm{~ms}$; conversion time $=40.96 \mathrm{~ms}$; temperature $=49 \mathrm{~K}$.

simulated spectrum that contains the same number of spectral features as a spectrum generated using an axial $\mathbf{g}$ matrix, but with line widths better simulated with the added rhombicity. Outer half-width at half-height line-width analysis of X-band and W-band cw EPR data was performed as described by Gaffney et al. ${ }^{28}$ The average line widths of the perpendicular turning points approximately double from X-band to W-band frequencies and those at the parallel turning points increase in width by a factor of $\sim 6$. Because the angular dependence of the resonance field is larger at $g_{z z}$ than at $g_{x x}$ and $g_{y y}, g$-strain could account for the observed line-width variation. At X-band, the line widths are presumably dominated by the unresolved hyperfine interactions of coupled protons and any strain that may exist in the hyperfine interaction. The well-understood $\mathrm{X}$-band EPR spectrum consists of eight powder patterns from the $2 I+1$ number of fully allowed $\Delta M_{I}=0$ EPR transitions, each influenced by the large hyperfine anisotropy and significant g anisotropy. As shown in Figure 1, these powder patterns are oriented with the parallel turning points occurring at a lower magnetic field than the corresponding perpendicular turning points for the $M_{I}=-7 / 2,-5 / 2,-3 / 2$ EPR transitions, and oriented with the parallel turning points occurring at a higher magnetic field positions than the corresponding perpendicular turning points for the $M_{I}=-1 / 2,1 / 2,3 / 2,5 / 2,7 / 2 \mathrm{EPR}$ transitions. The change in powder pattern orientation is a manifestation of the relative magnitudes of the elements of $\mathbf{A}$ 


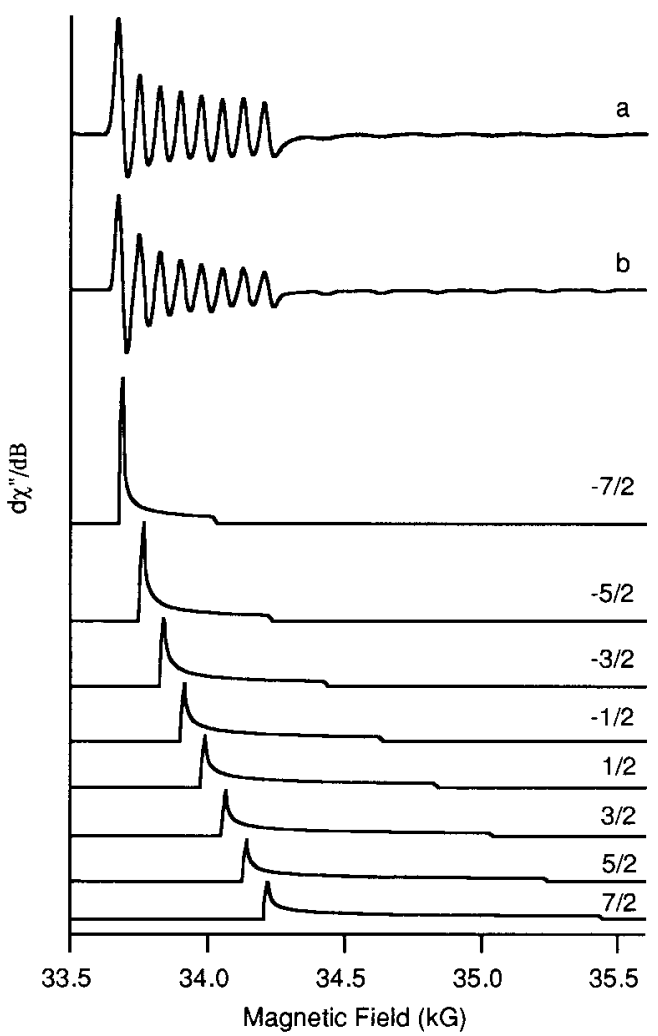

Figure 2. W-band EPR spectrum (a) of $\left[\mathrm{VO}\left(\mathrm{H}_{2} \mathrm{O}\right)_{5}\right]^{2+}$; trace (b) is the simulation performed by matrix diagonalization and incorporating $70 \mathrm{MHz}$ of strain in $g_{z z}$. The simulation is constructed from a collection of 19 simulations with the intensities weighted by a Gaussian distribution of $g_{z z}$ values. The additional traces show the eight individual $M_{I}=-7 / 2, \ldots,+7 / 2$ powder patterns. These traces are shown in absorption mode rather than as a derivative as in the cw-EPR spectrum for simplicity, and are generated for an axial $g$ tensor with the perpendicular value equal to the average of $g_{x x}$ and $g_{y y}$. Experimental conditions are as follows: $\mathrm{v}_{\mathrm{mw}}=94.007447 \mathrm{GHz}$; MW power $=\sim 50$ $\mathrm{nW}$; modulation amplitude $=15 \mathrm{G}$; time constant $=40.96 \mathrm{~ms}$; conversion time $=81.92 \mathrm{~ms}$; temperature $=20 \mathrm{~K}$.

and $\mathbf{g}$. The $M_{I}= \pm 7 / 2$ powder patterns do not overlap with any of the other EPR transitions at the parallel turning points and thus allow for absolute $M_{I}$ selection in ${ }^{51} \mathrm{~V}$ ENDOR experiments. ${ }^{29,30}$ The large spectral widths $(\sim 550$ and $\sim 400 \mathrm{G}$ wide, respectively) of these powder patterns allow for excellent orientation selection for ENDOR experiments performed at the parallel turning points of these two powder patterns. At W-band frequencies the spectrum greatly simplifies as the electron Zeeman interaction increases in magnitude by a factor of approximately 10 relative to the hyperfine interaction. This gives powder patterns all oriented with the perpendicular turning points occurring at a lower magnetic field value than the corresponding parallel turning points, and with little overlap of parallel and perpendicular features. These powder pattern breadths increase with $M_{I}$ because the anisotropy of the hyperfine interaction is still significant at $\mathrm{W}$-band frequencies.

ENDOR. Perturbation theory can be used to estimate $P_{\|}$by using eq 5 and fitting the ENDOR transitions observed at the $M_{I}= \pm 7 / 2$ parallel turning points at 4510 and $3120 \mathrm{G}$ (see Figure 3) of the EPR spectrum to Gaussian line shapes to determine the splitting frequency for each pair of transitions. The observed ENDOR transitions are shown in the upper and lower traces of Figure 3 . The perturbation theory calculated $P_{\|}$ value is $-0.129 \pm 0.005 \mathrm{MHz}$, with the reported error arising from the statistical error of the estimation of the splitting frequencies of the ENDOR transitions.

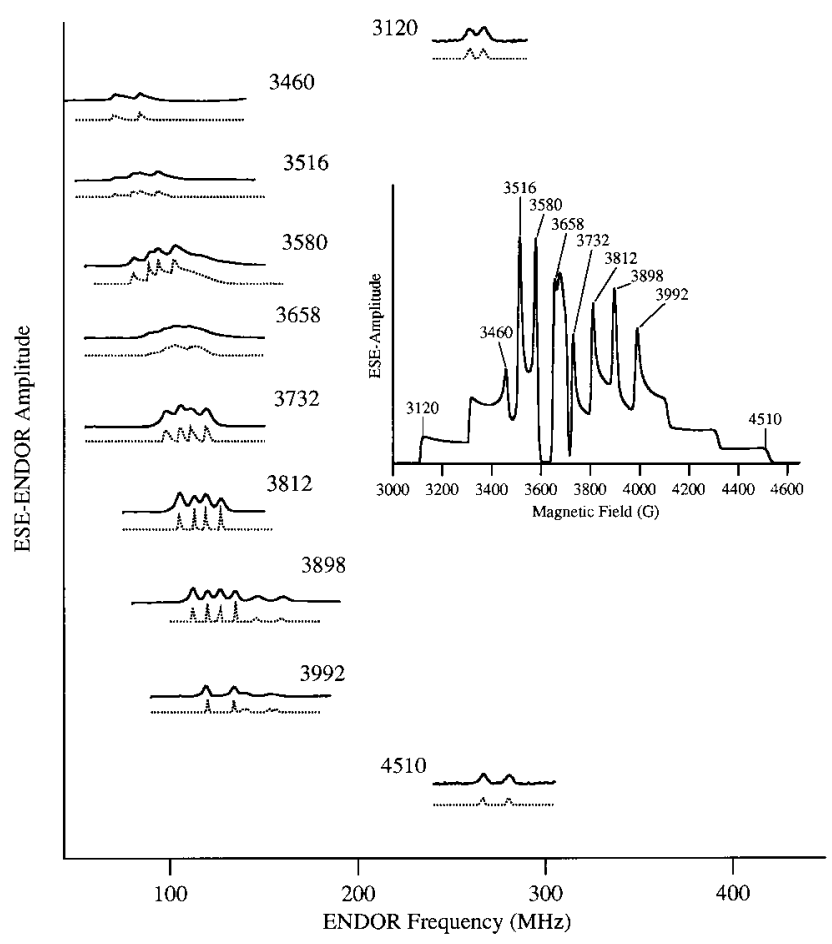

Figure 3. ESE-ENDOR of $\left[\mathrm{VO}\left(\mathrm{H}_{2} \mathrm{O}\right)_{5}\right]^{2+}$ at parallel and perpendicular turning points of the EPR spectrum. Field positions are indicated in the inset ESE magnetic field sweep spectrum. The dashed lines are simulations generated by diagonalization of the of the Hamiltonian of eq 1. Experimental conditions for the ENDOR data were as follows: $\mathrm{v}_{\mathrm{mw}}=10.339 \mathrm{GHz} ; \tau=500 \mathrm{~ns} ; \pi / 2 \mathrm{MW}$ pulse duration $=100 \mathrm{~ns} ; \pi$ MW pulse duration $=200 \mathrm{~ns} ;$ MW power $\sim 0.5 \mathrm{~W}$; ENDOR rf pulse duration $=18 \mu \mathrm{s} ; T=20 \mu \mathrm{s} ;$ rf power $\sim 100 \mathrm{~W}$. Experimental conditions for the ESE magnetic field sweep are as follows: $\mathrm{v}_{\mathrm{mw}}=$ $10.339 \mathrm{GHz} ; \tau=500 \mathrm{~ns} ; \pi / 2 \mathrm{MW}$ pulse duration $=100 \mathrm{~ns} ; \pi \mathrm{MW}$ pulse duration $=200 \mathrm{~ns}$; MW power $\sim 0.5 \mathrm{~W}$.

A more rigorous approach that we introduce here to the ENDOR analysis is the use of ENDOR simulations based on matrix diagonalization of the spin Hamiltonian (eq 1). The matrix diagonalization simulation of ENDOR data is performed at specific field values corresponding to the various parallel and perpendicular EPR turning points at which we obtained ENDOR spectra (Figure 3). The program calculates the ENDOR frequencies from only those of the 20000 molecular orientations for which the diagonalized Hamiltonian matrix gives an EPR transition within the specified bandwidth $(16 \mathrm{MHz})$ of the resonant frequency at a particular magnetic field value. Excellent simulations are obtained throughout the EPR spectrum with a $P_{\|}$value of $-0.20 \pm 0.01 \mathrm{MHz}$. The asymmetry parameter $\eta$ is estimated to be zero, but the frequencies of ENDOR transitions are not particularly sensitive to the asymmetry parameter. Values of $\eta$ ranging from 0 to 0.4 give ENDOR transitions at frequencies comparable to the experimental data, and as a result there is significant error in the experimental determination of $\eta$ by ${ }^{51} \mathrm{~V}$ ENDOR in this particular case.

We have performed test calculations in an effort to resolve the discrepancy between $P_{\|}$values obtained by perturbation theory and matrix diagonalization analysis. The difference in magnitudes of second-order perturbation theory estimates of $P_{\|}$ and those estimated by matrix diagonalization arises because of the lack of a third-order hyperfine term which is of significant magnitude when compared to the nuclear quadrupole interaction for typical vanadium hyperfine couplings. Additionally, the pulsed ENDOR experiments, due to finite microwave pulse bandwidth, do not select only those molecular orientations that 
are identically parallel to the external magnetic field, the ideal case for which the perturbation theory expressions have been derived. While the second-order perturbation theory estimates are useful for illustrating trends in the nuclear quadrupole coupling constants, the values can be more precisely calculated by the matrix diagonalization simulations of ENDOR data, which take the pulse bandwidth into account.

DFT Calculations. The nuclear quadrupole coupling constant $P_{\|}$can be theoretically determined from the DFT calculated electric field gradient. The Gaussian 94 output of the electric field gradient traceless tensor allows for the calculation of $e^{2} q Q$, from which $P_{\|}$may be calculated using eq 3 , and the asymmetry parameter $\eta$. The calculated bond lengths of the optimized structure of $\left[\mathrm{VO}\left(\mathrm{H}_{2} \mathrm{O}\right)_{5}\right]^{2+}\left(\mathrm{V}-\mathrm{O}\left(\mathrm{H}_{2} \mathrm{O}\right)\right.$ axial $=2.17 \AA$, $\mathrm{V}-\mathrm{O}\left(\mathrm{H}_{2} \mathrm{O}\right)$ equatorial $=2.03 \AA, \mathrm{V}-\mathrm{O}$ oxo $\left.=1.57 \AA\right)$ agree well with $\mathrm{X}$-ray crystallography results of the analogous VO$\mathrm{SO}_{4} \cdot 5 \mathrm{H}_{2} \mathrm{O}$ ion $\left(\mathrm{V}-\mathrm{O}\left(\mathrm{H}_{2} \mathrm{O}\right)\right.$ axial $=2.22 \AA, \mathrm{V}-\mathrm{O}\left(\mathrm{H}_{2} \mathrm{O}\right)$ equatorial $=2.04 \AA, \mathrm{V}-\mathrm{O}$ oxo $=1.60 \AA) .{ }^{31}$ The optimized geometry is somewhat distorted, with an average $\mathrm{O}$ (oxo) $-\mathrm{V}-$ $\mathrm{O}$ (equatorial $\mathrm{H}_{2} \mathrm{O}$ ) angle of $97.8^{\circ}$. The nuclear quadrupole coupling parameters from ab initio calculations are $P_{\|}=-0.185$ $\mathrm{MHz}$ and $\eta=0.325$, which compares well with the $P_{\|}$value determined by ENDOR simulation of $-0.20 \mathrm{MHz}$ for pentaaquaoxovanadium(IV). A calculation was also performed on the hypothetical case of the axially dehydrated tetraaquaoxovanadium(IV) ion. The molecular geometry was reoptimized and is best described as distorted square pyramidal with an average $\mathrm{O}($ oxo $)-\mathrm{V}-\mathrm{O}$ (equatorial $\mathrm{H}_{2} \mathrm{O}$ ) angle of $105.3^{\circ}$, yielding a $P_{\|}$value of $-0.299 \mathrm{MHz}$ and $\eta$ of 0.254 . The data from these two calculations follows the trend shown in our previous study of other oxovanadium(IV) molecules, such as bis(acetylacetonato)oxovanadium(IV) with a vacant axial binding site and a $P_{\|}$of $-0.9 \mathrm{MHz}$, compared to bis(acetylacetonato)oxovanadium(IV) coordinated axially by ethanol with a $P_{\|}$of $-0.5 \mathrm{MHz}$, where axial coordination significantly decreases the magnitude of $P_{\|}$(we note that these values were estimated by matrix diagonalization and differ from our previously published values determined by perturbation theory). ${ }^{9}$ Additional DFT calculations were performed on fixed geometry complexes with $\mathrm{O}($ oxo $)-\mathrm{V}-\mathrm{O}\left(\right.$ equatorial $\left.\mathrm{H}_{2} \mathrm{O}\right)$ angles constrained to $90^{\circ}$. For pentaaquaoxovanadium(IV) in the constrained geometry the nuclear quadrupole parameters $P_{\|}$and $\eta$ were calculated as $-0.297 \mathrm{MHz}$ and 0.191, respectively. For the constrained geometry model of tetraaquaoxovanadium(IV), $P_{\|}$and $\eta$ were calculated as $-0.388 \mathrm{MHz}$ and 0.124 , respectively. The above calculations on geometrically optimized complexes and model systems illustrate how molecular distortions can change the magnitude of the nuclear quadrupole coupling constant as the amount of electron density donated opposite the oxo bond changes as a function of the equatorial ligand geometry. Axial ligation also serves to greatly diminish the nuclear quadrupole coupling constant as electron density is donated opposite the oxo bond. Additional studies may also allow for structural data to be extracted from the asymmetry parameter, however these values are not easily determined experimentally.

\section{Conclusion}

Simulations of both W-band and X-band EPR spectra of [VO$\left.\left(\mathrm{H}_{2} \mathrm{O}\right)_{5}\right]^{2+}$ have been utilized to obtain accurate $\mathbf{g}$ and $\mathbf{A}$ matrix values. These are employed for matrix diagonalization simulation of ${ }^{51} \mathrm{~V}$ ENDOR data, providing an estimate of the nuclear quadrupole coupling constant, $P_{\|}=-0.20 \mathrm{MHz}\left(\left|e^{2} q Q\right|=5.6\right.$ $\mathrm{MHz}$ ). This value is compared to second order perturbation theory analysis which gives a value of $P_{\|}=-0.129 \mathrm{MHz}$. The difference in the magnitude of the quadrupole coupling constants calculated by the two methods is the result of a significant thirdorder hyperfine contribution and the error that occurs because the perturbation theory result does not account for the bandwidth of the EPR excitation pulses. We consider second-order perturbation theory analysis to be adequate only for describing the trends in the magnitude of the quadrupole coupling constants, but not for determining the absolute magnitude of $P_{\|}$.

The value of the nuclear quadrupole coupling constant obtained by ab initio density functional theory calculations $\left(P_{\|}=-0.185 \mathrm{MHz}\right)$ is in excellent agreement with the experimental value determined by matrix diagonalization simulation of ${ }^{51} \mathrm{~V}$ ENDOR data $\left(P_{\|}=-0.20 \pm 0.01 \mathrm{MHz}\right)$. This result and the additional calculations performed illustrate that axial ligands and equatorial ligand geometry can influence the magnitude of the nuclear quadrupole coupling constant. DFT is thus shown to be useful for the theoretical determination of these values in vanadyl complexes, and the combination of ENDOR, multifrequency EPR, and DFT provides a powerful structural tool for the study of vanadyl complexes.

Note Added in Proof. A recent EPR study at frequencies from 9 to $376 \mathrm{GHz}$ showed that the increase in line width of various features of the spectra of vanadyl in water/methanol was a linear function of microwave frequency. (Mustafi, D.; Galtseva, E. V.; Krzytek, J.; Brunel, L.-C.; Makinen, M. W. High-Frequency EPR Studies of $\mathrm{VO}^{2+}$ in Low Temperature Glasses, manuscript preprint.)

Acknowledgment. This work was supported by grants from the NIH (GM48242 to R.D.B. and GM36232 to B.J.G.). We thank Kristy A. Campbell for acquisition of the X-band cw EPR spectrum and her assistance in the acquisition of the W-band cw EPR data, and Dr. David W. Randall for sharing his expertise in ENDOR spectral simulations.

\section{References and Notes}

(1) Wever, R.; Krenn, B. E. In Vanadium in Biological Systems; Chasteen, N. D., Ed.; Kluwer Academic Publishers: Boston, MA, 1990; pp 81-98.

(2) Eady, R. R. In Vanadium in Biological Systems; Chasteen, N. D., Ed.; Kluwer Academic Publishers: Boston, MA, 1990; pp 99-128.

(3) Chasteen, N. D. In Biological Magnetic Resonance; Berliner, L. J., Reuben, J., Eds.; Plenum Press: New York, 1981; pp 53-119.

(4) Eaton, S. S.; Eaton G. R. In Vanadium in Biological Systems; Chasteen, N. D., Ed.; Kluwer Academic Publishers: Boston, MA, 1990; pp 199-222.

(5) Dubyak, G. R. Kleinzeller, A. J. Biol. Chem. 1980, 255, 5306.

(6) Shechter, Y.; Karlish, S. J. D. Nature 1980, 284, 556.

(7) Orvig, C.; Thompson, K. H.; Battel, M.; McNeal, J. H. In Vanadium Compounds as Insulin Mimics; Orvig, C., Thompson, K. H., Battel, M. McNeal, J. H., Eds.; Dekker: New York, 1995; Vol. 31, pp 575-594.

(8) Cornman, C. R.; Geiser-Bush, K. M.; Rowley, S. P.; Boyle, P. D. Inorg. Chem. 1997, 36, 6401.

(9) Grant, C. V.; Ball, J. A.; Hamstra, B. J.; Pecoraro, V. L.; Britt, R. D. J. Phys. Chem. B 1998, 102, 8145 .

(10) Van Willigen, H. J. J. Magn. Reson. 1980, 39, 37.

(11) Van Willigen, H. J.; Mulks, C. F.; Atherton, N. M. Inorg. Chem 1982, 21, 1708

(12) Strach, S. J.; Bramley, R. Chem. Phys. Lett. 1984, 109, 363.

(13) Borcherts, R. H.; Kikuchi, C. J. Chem. Phys. 1964, 40, 2270

(14) Kohin, R. P. Magn. Reson. Rev. 1979, 5, 75.

(15) Atherton, N. M.; Shackleton, J. F. Mol. Phys. 1980, 39, 1471.

(16) Misra, S. K.; Sun, J. Phys Rev. B. 1990, 42, 8601.

(17) Suryanarayana, D.; Narayana, P. A.; Kevan, L. J. J. Phys. Chem. 1982, 86,4579 .

(18) Dikanov, S. A.; Yudanov, V. F.; Tsvetkov, Yu. D. J. Magn. Reson. 1979, 34, 631

(19) Mustafi, D.; Makinen, M. W. Inorg. Chem. 1988, 27, 3360.

(20) Sturgeon, B. E.; Britt, R. D. Rev. Sci. Instrum. 1992, 63, 2187.

(21) Sturgeon, B. E.; Ball, J. A.; Randall, D. W.; Britt, R. D. J. Phys. Chem. 1994, 98, 12871.

(22) Davies, E. R. Phys. Lett. 1974, 47A, 1. 
(23) Hoffman, B. M.; DeRose, V. J.; Doan, P. E.; Gurbiel, R. J.; Houseman, A. L. P.; Telser, J. In Biological Magnetic Resonance; Berliner, I. J., Reuben, J., Eds.; Plenum: New York, 1993; Vol. 13, pp 151-218. (24) Frish, M. J.; Trucks, G. W.; Schlegel, H. B.; Gill, P. M. W.; Johnson, B. G.; Robb, M. A.; Cheeseman, J. R.; Keith, T. A.; Petersson, G. A.; Montgomery, J. A.; Raghavachari, K.; Al-Laham, M. A.; Zakrzewski, V. G.; Oritz, J. V.; Foresman, J. B.; Cioslowski, J.; Stefanov, B. B.; Nanayakkara, A.; Challacombe, M.; Peng, C. Y.; Ayala, P. Y.; Chen, W. Wong, M. W.; Andres, J. L.; Replogle, E. S.; Gomperts, R.; Martin, R. L.; Fox, D. J.; Blinkley, J. S.; Defrees, D. J.; Baker, J.; Stewart, J. P.; HeadGordon, M.; Gonzalez, C.; Pople, J. A. Gaussian-94; Gaussian, Inc.: Pittsburgh, PA, 1995.

(25) Rasolov, V. A.; Pople, J. A.; Ratner, M. A.; Windus, T. L. J. Chem. Phys. 1998, 109, 1223.
(26) Abragam, A.; Bleaney, B. Electron Paramagnetic Resonance of Transition Ions; Dover Publications: New York, 1986; pp 166-167.

(27) Weltner, W., Jr. Magnetic Atoms and Molecules; Dover; New York, 1983; pp 342.

(28) Gaffney, B. J.; Maguire, B. C.; Weber, R. T.; Maresch, G. G. Appl. Magn. Reson. 1999, 16, 207.

(29) Randall, D. W.; Sturgeon, B. E.; Lorigan, G. A.; Chan, M. K.; Klein, M. P.; Armstrong, W. H.; Britt, R. D. J. Am. Chem. Soc. 1995, 117 11780.

(30) Randall, D. W.; Chan, M. K.; Armstrong, W. H.; Britt, R. D. Mol. Phys. 1998, 95, 1283.

(31) Ballhausen, C. J.; Djurinskij, B. F.; Watson, K. J. J. Am. Chem. Soc. 1968, 90, 3305. 